\title{
Use of smart glasses for ultrasound- guided peripheral venous access: a randomized controlled pilot study
}

elSSN: $2383-4625$

\author{
Hyunmook Lim', Min Joung Kim², Joon Min Park', Kyung Hwan Kim', \\ Junseok Park', Dong Wun Shin', Hoon Kim', Woochan Jeon', \\ Hyunjong Kim ${ }^{1}$, Jungeon Kim ${ }^{1}$
}

\begin{abstract}
'Department of Emergency Medicine, Inje University Ilsan Paik Hospital, Goyang, Korea
${ }^{2}$ Department of Emergency Medicine, Yonsei University College of Medicine, Seoul, Korea
\end{abstract}

Objective Smart glasses can provide sonographers with real-time ultrasound images. In the present study, we aimed to evaluate the utility of smart-glasses for ultrasound-guided peripheral venous access.

Methods In this randomized, crossover-design, simulation study, 12 participants were recruited from the emergency department residents at a university hospital. Each participant attempted ultrasound-guided peripheral venous access on a pediatric phantom at intervals of 5 days with (glasses group) or without (non-glasses group) the use of smart glasses. In the glasses group, participants confirmed the ultrasound image through the lens of the smart glasses. In the nonglasses group, participants confirmed the ultrasound image through the display viewer located next to the phantom. Procedure time was regarded as the primary outcome, while secondary outcomes included the number of head movements for the participant, number of skin punctures, number of needle redirections, and subjective difficulty.

Results No significant differences in procedural time were observed between the groups (nonglasses group: median time, 15.5 seconds; interquartile range [IQR], 10.3 to 27.3 seconds; glasses group: median time, 19.0 seconds; IQR, 14.3 to 39.3 seconds; $P=0.58$ ). The number of head movements was lower in the glasses group than in the non-glasses group (glasses group: median, $0 ; I Q R, 0$ to 0 ; non-glasses group: median, 4; IQR, 3 to 5; $P<0.01)$. No significant differences in the number of skin punctures or needle restrictions were observed between the groups.

Conclusion Our results indicate that smart-glasses may aid in ensuring ultrasound-guided peripheral venous access by reducing head movements.

Keywords Wearable electronic devices; Ultrasonography; Wireless technology

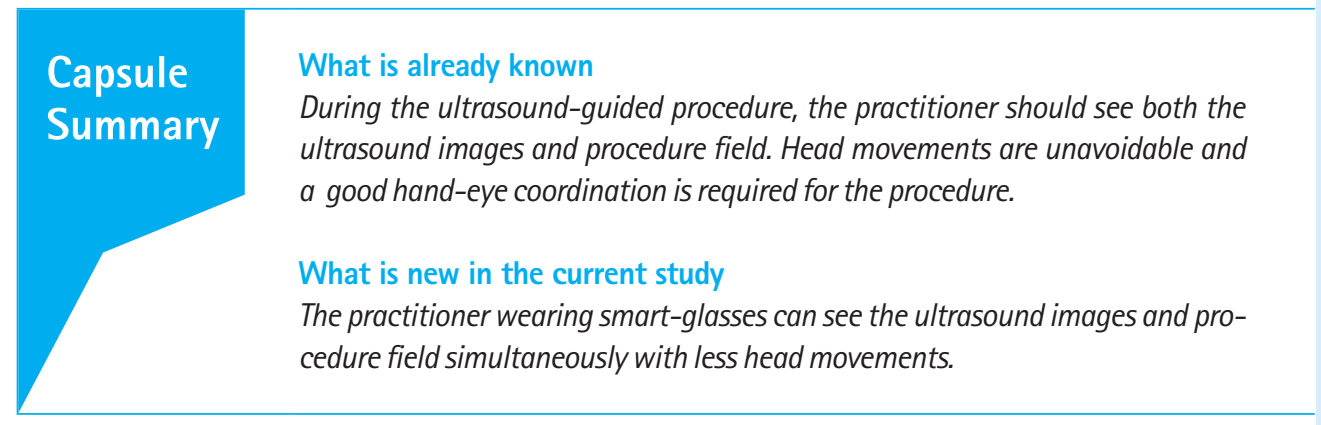

\section{Received: 28 March 2019 \\ Revised: 24 April 2019 \\ Accepted: 29 April 2019}

Correspondence to: Joon Min Park Department of Emergency Medicine, Inje University Ilsan Paik Hospital, 170 Juhwa-ro, Ilsanseo-gu, Goyang 10380, Korea

E-mail:aero7@paik.ac.kr ORCID

http://orcid.org/0000-0001-7258-8892

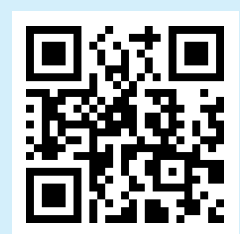

How to cite this article:

Lim H, Kim MJ, Park JM, Kim KH, Park J, Shin DW, Kim H, Jeon W, Kim H, Kim J. Use of smart glasses for ultrasound-guided peripheral venous access: a randomized controlled pilot study. Clin Exp Emerg Med 2019;6(4):356-361.

This is an Open Access article distributed under the terms of the Creative Commons Attribution Non-Commercial License (http:// creativecommons.org/licenses/by-nc/4.0/). 


\section{INTRODUCTION}

Peripheral venous access is required for various clinical purposes including blood sampling for laboratory tests and administration of intravenous drugs or fluids. In pediatric patients, securing a peripheral venous route is frequently difficult because their vessels are thin and run relatively deep. In addition, children may not cooperate, impeding successful access in clinical situations. ${ }^{1}$ Thus, several research groups have examined the efficacy of assistive technologies for peripheral venous access, such as transilluminators, near-infrared light devices, and ultrasonography. ${ }^{2-4}$

Ultrasound aids practitioners in locating and confirming a target vessel in real-time during venous access procedures. Previous studies have reported that the use of ultrasound is associated with a higher success rate on the first attempt, fewer total attempts, and shorter procedure time in securing venous access in pediatric patients. ${ }^{4,5}$ Conventional ultrasound is performed at the side of the patient's bed, and practitioners must rotate their heads to confirm the real-time image on the display monitor. This head movement theoretically hampers the hand-eye coordination required for successful ultrasound-guided venous access. ${ }^{6}$ The development of wireless ultrasound machines allows the display monitor (tablet computer or specific display monitor) to be placed closer to the practitioner (e.g., next to the patient on the bed).

A previous study investigated the efficacy of wearable smart glasses (Google Glass; Google, Mountain View, CA, USA) for displaying real-time ultrasound images during central venous access in a simulated setting. In that study, confirming the image through a display attached to the glasses reduced head movement, relative to that required for conventional ultrasound. Because peripheral veins are thinner than central veins, especially in children, frequent head movement to confirm the ultrasound image may impede successful venous access in this population. However, no previous studies have explored the usefulness of smart glasses for ultrasound-guided peripheral venous access. Thus, in the present study, we investigated the practicality of smart glasses for peripheral venous access in pediatric patients in a simulated setting.

\section{METHODS}

\section{Study design and setting}

This prospective, randomized, crossover pilot study was conducted in a simulation room at a tertiary hospital in Korea from June 20 to July 20, 2018. The ethics committee of the hospital approved the protocol of the study (2018-04-013), which was registered in ClinicalTrials.gov (NCT03565419) and conformed to the tenets of the Declaration of Helsinki.

\section{Study participants}

We recruited volunteers by sending e-mails to residents (PGY2PGY5) of the emergency department at a tertiary university hospital. All volunteers had already completed a 2-hour education course regarding the ultrasound-guided procedure and had experience with ultrasound-guided venous access ( $\geq 5$ times). The primary investigator explained the purpose of the study and the overall protocol to all volunteers, following which written informed consent was obtained from those who agreed to enroll.

\section{Study protocol}

First, the details of the simulation protocol were explained to all participants by an investigator. Then, because none of the participants had used smart glasses for confirming ultrasound images or had experience with simulated phantoms, each participant practiced ultrasound-guided venous access with and without smart glasses for at least 30 minutes before participating in the simulation. A phantom simulating adult vessels (Branched 2 vessel Ultrasound Training Block model; CAE Healthcare, Sarasota, $\mathrm{FL}, \mathrm{USA}$ ) was used for these practice sessions. A wireless SONON 300L ultrasound machine (Healcerion, Seoul, Korea) and Moverio BT-300 smart glasses (Epson, Nagano, Japan) were used for the simulations (Fig. 1). The binocular Moverio smart glasses were equipped with a small OLED (organic light-emitting diode) display, which allows practitioners to see the background and displayed image simultaneously (Fig. 2). The real-time ultrasound image was first transmitted to a tablet computer (Galaxy Tab; Samsung, Seoul, Korea) via Wi-Fi, following which the image was transmitted to the smart glasses via Miracast. Although this process proceeded simultaneously, there was a slight time delay between the display of images on the tablet and smart glasses $(<1$ second). When the smart glasses were not used, the real-time ultrasound image was displayed on a tablet computer located next to the phantom (left or right side, based on participant's prefer-

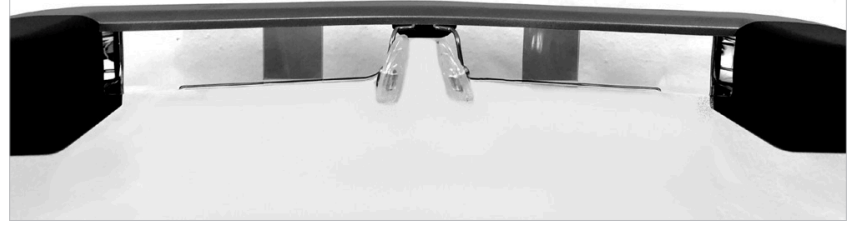

Fig. 1. The smart glasses (Moverio BT-300) from the wearer's perspective. Two OLED (organic light-emitting diode) lenses that display images are located at the center of the glasses. 


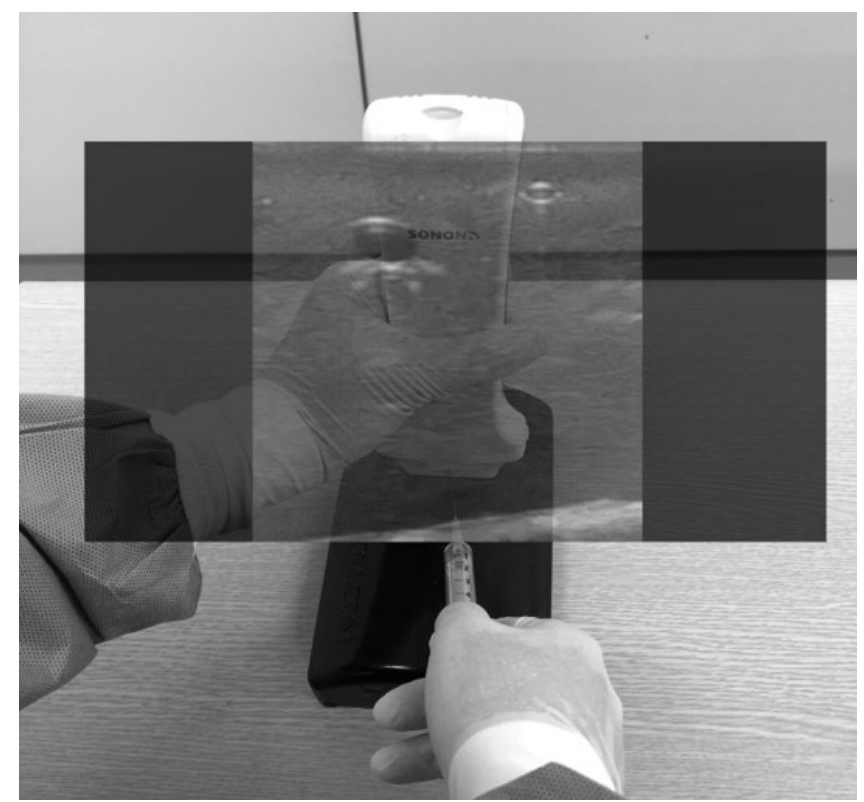

Fig. 2. Example of an ultrasound image displayed on the viewer of the smart glasses during the procedure.

ence). On the day following the practice sessions, each participant took part in one of two simulated ultrasound-guided venous access scenarios: with (glasses group) or without (non-glasses group) the use of smart glasses (Fig. 3). Given that ultrasound is mainly used for venous access under difficult conditions, a phantom simulating pediatric vessels (Branched 4 vessel Ultrasound Training Block model, CAE Healthcare) was used in the simulated scenario. After a 5-day washout period, each participant engaged in another simulated scenario.

A 20-gauge needle attached to a $10-\mathrm{mL}$ syringe was used for venous access. All ultrasound-guided venous access procedures were performed using a short-axis approach. A researcher supervised the entire simulated scenario and confirmed successful venous access, which was defined as confirmed blood aspiration in the syringe. Each simulated scenario was recorded on video. The order of each participant's scenarios was determined via blocked randomization using random permuted blocks. Sealed radiopaque envelopes were used for the blinded allocation. The randomization process was implemented by an investigator.

\section{Data collection and statistical analysis}

A researcher blinded to the study objective measured most study outcomes by reviewing the recorded videos. Procedure time in seconds was regarded as the primary outcome. Procedure time was defined as the time from the application of the ultrasound probe on the phantom to successful venous access. Secondary outcomes included the number of head movements until suc-
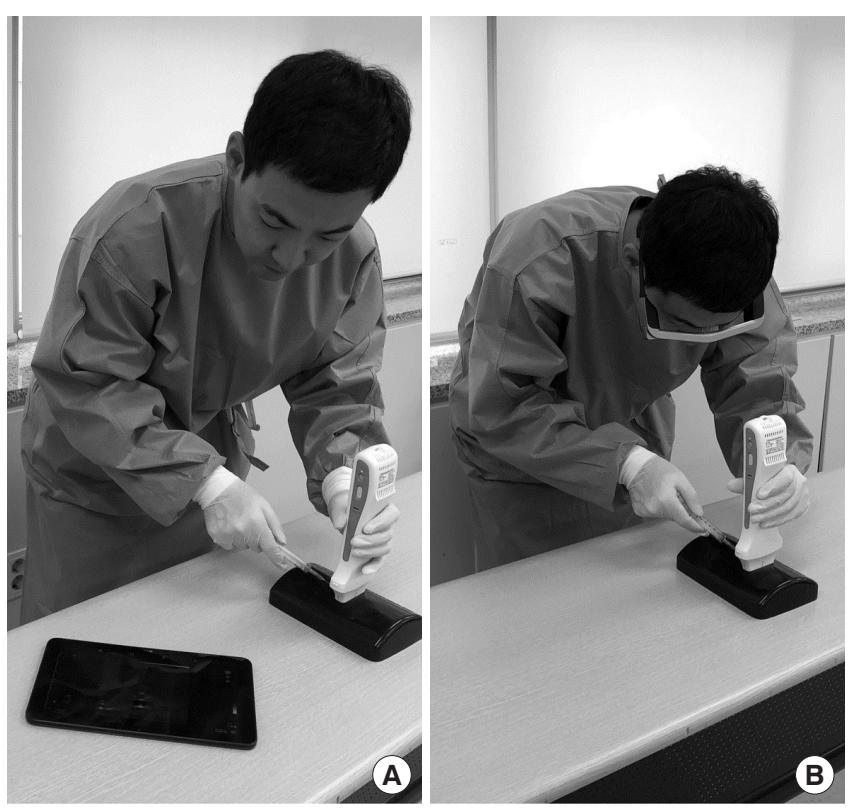

Fig. 3. Ultrasound-guided procedures in both groups. (A) Non-glasses group and (B) glasses group. The consent to use the picture was acquired from the participant in the picture.

cessful venous access, number of skin punctures until successful venous access, number of needle redirections until successful venous access, and subjective difficulty of the procedure based on a visual analogue scale (VAS) of 0 to 100 (0, easiest; 100, hardest). Participants reported subjective difficulty ratings after each simulation scenario.

Based on a previous report, we determined that the sample for the pilot study should include a minimum of 12 participants in both the control and intervention arms. ${ }^{8}$ Outcome variables were described using medians and interquartile ranges (IQRs). MannWhitney U-tests were used to compare outcomes between the two arms. IBM SPSS Statistics ver. 21 (IBM, Armonk, NY, USA) was used for statistical analysis, and P-values $<0.05$ were considered statistically significant.

\section{RESULTS}

Twelve volunteers were enrolled in our study, none of whom dropped out (Fig. 4). All participants were men, and the median age was 32 years. There were three participants for each grade (PGY2-PGY5).

No significant differences in procedure time were observed between the glasses and non-glasses groups (Table 1 and Fig. 5). However, the number of head movements was significantly higher in the non-glasses group than in the glasses group. No significant differences in the number of skin punctures or needle redirections were observed between the groups (Table 1). Participants in the 


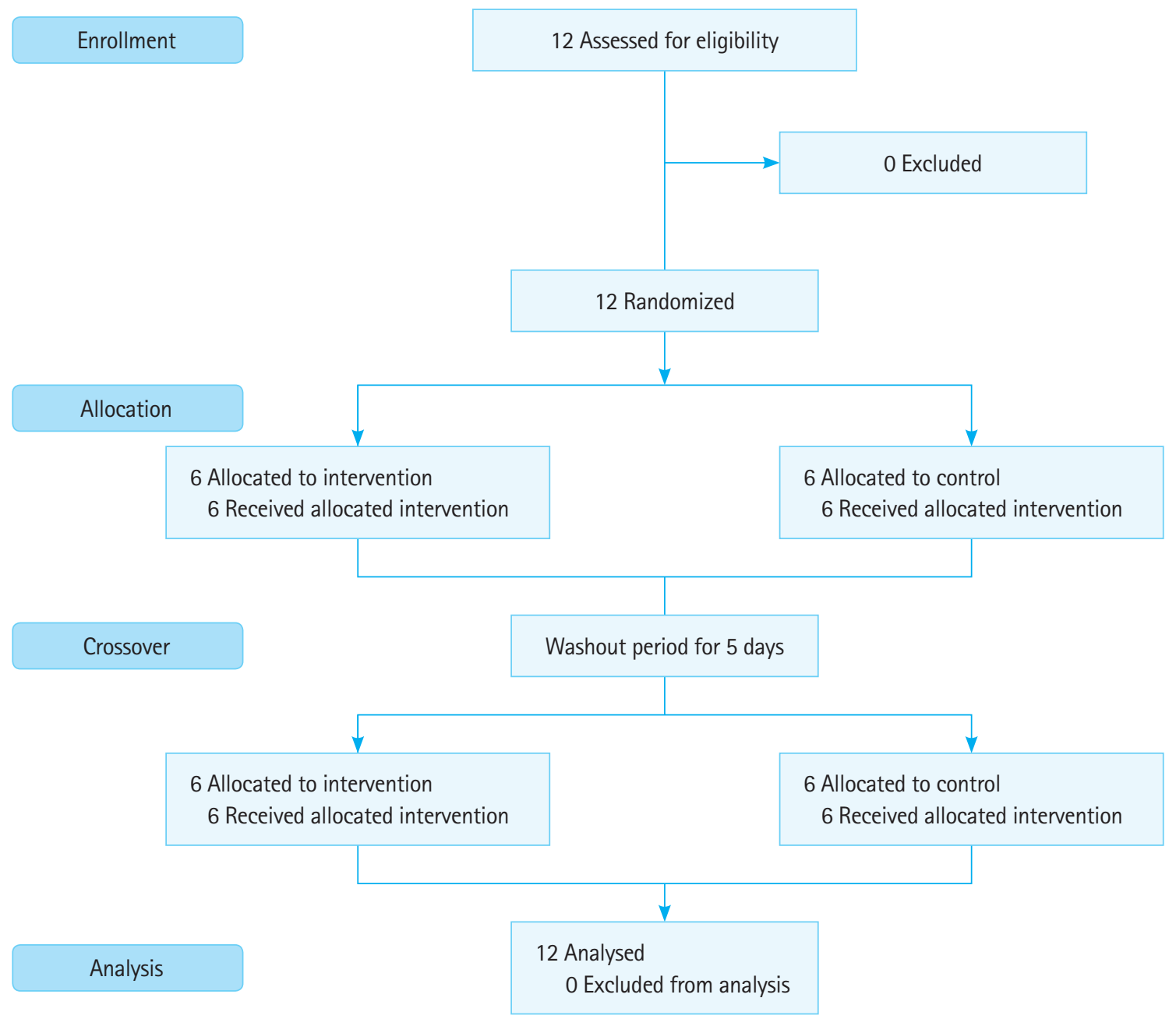

Fig. 4. Flow diagram of the study.

Table 1. Procedure time, number of head movement, skin puncture, and needle redirection in both groups

\begin{tabular}{lccc}
\hline & Non-glasses group & Glasses group & P-value \\
\hline Procedure time (sec) & $15.5(10.3-27.3)$ & $19.0(14.3-39.3)$ & 0.58 \\
No. of head movements & $4(3-5)$ & $0(0-0)$ & $<0.01$ \\
No. of skin punctures & $1(1-1)$ & $1(1-1)$ & 0.99 \\
No. of needle redirections & $0(0-3)$ & $0.5(0-2)$ & 0.55 \\
\hline
\end{tabular}

Values are presented as median (interquartile range).

glasses group reported greater subjective difficulty than those in the non-glasses group (glasses group: median VAS, 30; IQR, 20 to 65; non-glasses group: median VAS, 15; IQR, 0 to $30 ; P=0.04$ ).

\section{DISCUSSION}

The application of bedside ultrasound has enabled not only faster and more accurate diagnoses but also safer medical practices, providing practitioners with real-time ultrasound images. Re-

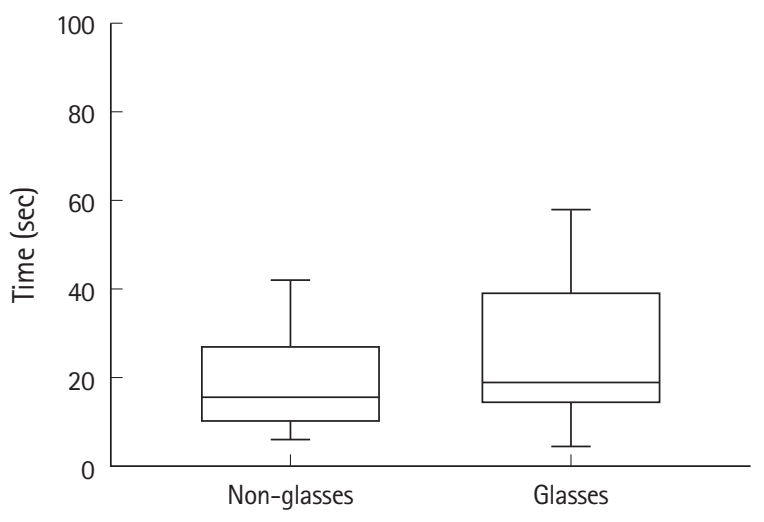

Fig. 5. Comparison of procedure time of the 2 group $(\mathrm{P}=0.575)$.

cently, small, portable ultrasound machines and wireless devices have been developed for use in emergent or urgent situations. ${ }^{9}$ Theoretically, wireless mobile ultrasound machines may aid in aseptic ultrasound-guided procedures, as wires connected to the ultrasound probe can interfere with the procedure. Although 
wireless mobile ultrasound is advantageous in many respects, it is still necessary to confirm the real-time image using an independent viewing device. Hence, in the present study, we investigated the utility of a wearable viewing device that displays real-time ultrasound images from wireless ultrasound during peripheral venous access procedures.

In our study, the use of smart glasses did not significantly increase procedure time, despite the users' relative lack of familiarity with the smart glasses. Previously, Wu et al.' reported that the time required for successful central venous access was longer when wearing smart glasses across all four training levels, with statistical significance in two of four levels. Such differences may have been due to differences in proficiency during the target procedure and a lack of familiarity with smart glasses. In the study by Wu et al., ${ }^{7}$ participants lacked experience in central venous access, and half of all participants were medical students. In our study, all participants had already performed peripheral venous access more than 100 times and ultrasound-guided venous access at least five times during their clinical practice period (minimum of 1.5 years). In addition, although we offered practice time to participants before participating in the simulation scenario, participants in the Wu et al. ${ }^{7}$ study wore smart glasses for the first time in the simulation scenario. Considering that the use of ultrasound decreases the time required for venous access, especially in difficult populations, our results suggest that smart glasses can aid in ensuring ultrasound-guided venous access without significant increases in procedure time. ${ }^{4,5}$

Prior to the simulation experiments, we assumed that confirming the real-time ultrasound image and the procedure fields simultaneously in the direct line of sight may aid in ultrasoundguided venous access because the participant would not need to move his head to confirm the image. Moving the head may lead to changes in depth or direction of needle insertion, as the procedure field is out of sight during head movement. In our study, participants made fewer head movements when wearing smart glasses than when not. This result is consistent with the findings of Wu et al., ${ }^{7}$ who reported no head movement in the Google Glass group yet several head movements among participants in the non-Google Glass group. The location of the display differed between our smart glasses and the Google Glass system used by Wu et al. ${ }^{7}$ While the image is displayed in the center of the practitioner's line of sight in our system, it is displayed in the upper right corner of the practitioner's line of sight in the Google Glass system. Although we did not compare the convenience of these two systems, we believe that images displayed in the center of the line sight may be more advantageous for ultrasound-guided venous access.
In clinical settings, more frequent skin punctures or needle redirections increase not only the patient's pain and anxiety but also the practitioner's level of stress, which are closely linked to patient/practitioner satisfaction and the level of rapport between the two parties. ${ }^{10}$ In our study, additional skin punctures and needle redirections were not required in most cases, and there were no significant differences between the two groups. Although Wu et al. ${ }^{7}$ reported that the number of needle redirections was higher among participants using Google Glass than those not using the system, this difference was not statistically significant. Nonetheless, such differences may have been due to a lack of proficiency in ultrasound-guided central venous access and unfamiliarity with the Google Glass system in the previous study.

In our study, the degree of subjective difficulty was slightly higher in the glasses group. Difficulty ratings were likely based on low familiarity with smart glasses despite practice time and an effort to adjust to the time delay between displays. Participants reported that smart glasses were helpful in acquiring the procedure field while keeping the ultrasound image in the line of sight. In the future, we expect these subjective difficulty ratings to decrease as the time delay between the display of the smart glasses and the real-time ultrasound image decreases, and as familiarity with smart glasses increases due to more widespread use in clinical environments.

The present study possesses some limitations of note, including its small sample size, which was not predetermined for securing statistical significance. However, as we investigated the feasibility of smart glasses for ultrasound-guided peripheral venous access among trained practitioners, our results highlight the need for further studies of their efficacy in various clinical settings. Second, neither the participants nor the researcher who controlled the actual simulation could be blinded, which may have resulted in performance bias. Third, considering that our study was conducted in a simulated setting, we were unable to examine the utility of smart glasses in actual practice. Future studies should investigate the efficacy of smart glasses for ultrasound-guided venous access in live patients.

In conclusion, in this pilot study, we observed no significant differences in the time required for successful ultrasound-guided peripheral venous access based on the use of smart glasses. However, fewer head movements were required when using smart glasses. Given these benefits, smart glasses may be particularly suitable for emergency situations, especially when used in conjunction with tools such as wireless ultrasound. In the future, prospective controlled trials are required to more fully elucidate the utility of smart glasses for ultrasound-guided peripheral venous access. 


\section{CONFLICT OF INTEREST}

No potential conflict of interest relevant to this article was reported.

\section{ACKNOWLEDGMENTS}

This work was supported by a National Research Foundation of Korea (NRF) grant funded by the Korean government (Ministry of Science, ICT \& Future Planning) (2017R1C1B5076798).

\section{REFERENCES}

1. Park JM, Kim MJ, Yim HW, Lee WC, Jeong H, Kim NJ. Utility of near-infrared light devices for pediatric peripheral intravenous cannulation: a systematic review and meta-analysis. Eur J Pediatr 2016;175:1975-88.

2. Hosokawa K, Kato H, Kishi C, Kato Y, Shime N. Transillumination by light-emitting diode facilitates peripheral venous cannulations in infants and small children. Acta Anaesthesiol Scand 2010;54:957-61.

3. Kim MJ, Park JM, Rhee N, et al. Efficacy of VeinViewer in pediatric peripheral intravenous access: a randomized controlled trial. Eur J Pediatr 2012;171:1121-5.
4. Doniger SJ, Ishimine P, Fox JC, Kanegaye JT. Randomized controlled trial of ultrasound-guided peripheral intravenous catheter placement versus traditional techniques in difficultaccess pediatric patients. Pediatr Emerg Care 2009;25:154-9.

5. Benkhadra M, Collignon M, Fournel I, et al. Ultrasound guidance allows faster peripheral IV cannulation in children under 3 years of age with difficult venous access: a prospective randomized study. Paediatr Anaesth 2012;22:449-54.

6. Weiner MM, Geldard P, Mittnacht AJ. Ultrasound-guided vascular access: a comprehensive review. J Cardiothorac Vasc Anesth 2013;27:345-60.

7. Wu TS, Dameff CJ, Tully JL. Ultrasound-guided central venous access using Google Glass. J Emerg Med 2014;47:668-75.

8. Julious SA. Sample size of 12 per group rule of thumb for a pilot study. Pharm Stat 2005;4:287-91.

9. Chen $Y$, Zheng $H$, Zang Z, Hong $X$, Cai $W$, Fang $Y$. Real-time ultrasound-guided percutaneous nephrolithotomy using newly developed wireless portable ultrasound: a single-center experience. Surg Innov 2018;25:333-8.

10. Heinrichs J, Fritze Z, Vandermeer B, Klassen T, Curtis S. Ultrasonographically guided peripheral intravenous cannulation of children and adults: a systematic review and meta-analysis. Ann Emerg Med 2013;61:444-54. 